\title{
Persönliches
}

\section{Prof. Dr. Erik Hägglund †}

Am 13. Mär\% ist Herr Prof. Dr. Erik Hä gg glund nach limgerer Krankheit, jedoch villig mernarlet rerschieden. Fir ik Hä golund, der neben Poler Klason der schwedischen Holz- und Zellstoflchemie zu ihrem Weltruf verloblfen hat, war nich! nur ein ungew ihlnlich origineller und produktiver Wissenshafiler, sondern er kannte wie sonst heiner die wirlilichen Probleme und Fintwicklungsmöghtichkeiten der Zellstoftwerke und der holwehemischen Industrie.

Bis zuletzt befabte er sich noch mit weitreichenden Arbeitsplänen. Sein Wirlien als l'orscher und I ehrer, als Förderer der
Industrje und als Organisator wurde anliallich seines siebrigsten Geburtstages von Prof. ENich Adler in dieser Zeil-

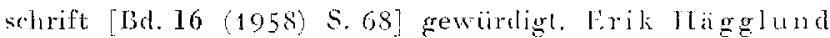
zeichnete nicht sur als mitwirkent an der Zeitschrilt , Hol als Poh- und Werkstolf", sondern er hat in ihe wiederholt bedeutende Arbeiten veröflentlichl. Der grobe Verhus schunerz nicht nur in Schweden, sondom iiborall in der Wolt scine Bewumberer und Freunde.

F. Rollmann Minuchen:

\section{Kleine Mitteilungen}

\section{Symposium über Biochemie des Holzes vom 1. bis 6.9.1958}

Der erste Tag des Symposiums iiher "Biochemie des Holzes", das im Rahmen des [V. Internationalen Kongresses fiit Biochemie vom 1. bis 6. September 1958 in Wien stall. fand und das von K. Kratzl (Wien) geleitet wurde, war den niedermolekularen nazessorischen Bestandteilen des Holzes

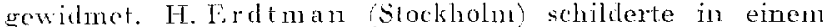
umfasenden Vortra iber ,Conifer Chemistry and Taxonomy of Conifers: ${ }^{\circ}$ die ungeheure Mamnigfaltiglieit der Inhaltsstolfe des Kernholzes der Coniferen. Zahhreiche Coniforenarten wurden auf viele defhnierte niedernolekulare Inhaltsstolfe untersucht, wobei neben der Isolierung auch papierchomalographisclie Verfahren herangezogen worden. Die Zuordnung der gefundenon Verbindungen auf die eirqeher Genera, Subgenera, Sectiones, Subsectiones und Species emö̈glicht eine chemische Tramomie und stellt eine wertvolle Ergänzung der botanischen Taxonomic dar. Dies um so mehr; als cs heute kein begrindetes System der Phylorenese der Pflanzen gibt und man mit einem mehr oder minder natürlichen Sistem arbeiten mub. Sohlieblich er wiihnte der Vortragende, dab gerade dic Chemie, cine bisher der Taxonomie sehr abseits stehende Wissenschaft, nicht nur unlerstiilzend wirken kimn, sondern anch neue Problene aufwirft.

W. Sandermann (Hanburg-Reinbek) Jielt einen Vortrat uber "Die Biogenese der Terpene nud "Terpenoide". Ausgehend von Wallachs "lsoprenhypothese" wurde das allgemeine Aufbauprinzip dieser Substanzliasse belandeli. burch Anwendung der Warkirungsterhnik liomnte gezeigl werden, dab Yssigsäure nach einem definierten Schema in Ferpene und Terpenoide eingebaut wird. In jüngster Zeit. bonnte in der Nevalonsäure $(\beta, \delta$-D)ilayldoxy- $\beta$-methylvaleriansïure) eine ummitlelbare Vorstufe gefunden werden, die nach Dekarboxvlierun in den Isoprenbaustein übergehı. Die als Vorstufe ebenfalls sehr wirlssame $\beta, \beta$-Dimethylacrylsüure liegt in einem Seitenzweig cles Biogeneseschemas. Die Möglichlseiten der Bilkhung der Sesqui- und Diterpene, die Ringschlüsse und Umlagerungen an diesen Grundlö̈rpern sowie die Biosynthese des Squalens und Cholesterins wurden mit hesondeser Beruchsichtigung der räumlichen Strultur crörtert und übersichtlich formuliert. Schlieblich berichtete der Vortzagende äber eigene Arbeiten zur Biogenese des Pulegons (bei Mentha pulegium L.) und der Abietin- und Dextropimarsäure (bei der kiefer), wobei ${ }^{24} \mathrm{C}$-markierte

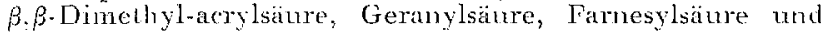
Geranylgeraniumsäure Verwendumg fanden.

In der anschliebenden Diskussion berichtete F. G. Stanley (Berkeley, USA) ebenlalls über Versuche zur Biogenese der T'erpene, wobei die Arbeiten von W. Sandermann ergänzt. werden konnten. Ilierbei hatten sich vowiegend gaschromatographische Trenumgen der Terpengemische bewährt.

H. Grisebacli (Freiburg i. Br.) sprach iiber, Die Biosynthese der Anthoryane". Die Bildung des Cyanidins wurde an liotholulkcimlingen tulersucht. Es lag hier der interessinte Fill vor, dals bei der Biosynthese des Flavanskeletts ron der Pllanze zwer verschiedene Wege der Aromatisierung beschritten werden. L-Phenylalanin- $\left.{ }^{14} \mathrm{C}\right)$ wurle nur in den Ring 13 des Cyanidins eingebaut. Dieser Ring, der nach einem Abbau als Protocatechusiure erthalten wird, scheint demnach auf dem Weg Zucker $\rightarrow$ Shikimisäure $\rightarrow$ Phenylbrenztraubensäure gebildet zu werden. Hingegen wird der
Ming A, dor Aromat des Chromangeriists, der now einem Abbau als Plylonglucin anfällt, aus Lssigsiature anfgebaut. Die Carboxylerruppe der Essigsaiure bildet jene (- Atome des Phlorortucins, welche Ifydroxylgruppen tagen. Wahrend die restlichen drei C-Aiome der Methylgruppe der lissigsioure enlstammen. Dieses Frrobnis komnte millels Parallelver-

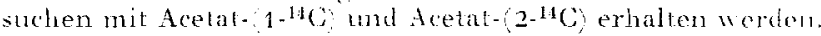
Coniferin, der Vorlitufor des Phonylpropansteletts des Limnins. und Meso-inosit, dem oft eine [Rolle als Bindeglied as ischan aliphatischen und aromalischen Verbindungen zugesproshen wurde, waren als Vorstufen zur Synthese des Cyanidins nuwirksan.

Der Diskussionsbeitrag von H. Kennik (Hejdclherg) wrmainzte die vorhergehenden Ansiburmgen. Auch bei dent Flavon Quercetin stammt der Ring B alls einet Vorstule mit Phenylpropansieledt. Dor Vortragende wies daranf hin, dabs min Lignin-, liavon- unt Anthorsan-Biossnthese nicht als isolierte Prozesse betrachten diufe und erwathe in diesem Zusanmenhang die zentrale Slellung der Zimisäuren bej dep phanalichen Exkretion.

An zweiten lagr des Symposiums wurle mit dere thhathdlung der hoclumolekularen Bestandecile des tholaes ho. monnen. A. Greatlonse Gainesrille, Gstj brarhle einen Fortrag ,On the Enamic Polysacharide Symthesis". Au

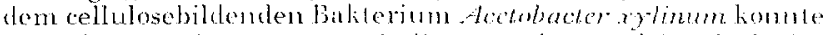

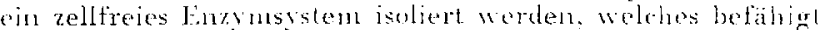

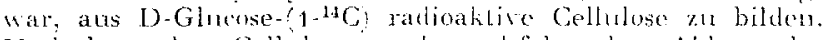
Hydrolyse der Collulose und nachfolgender Abbau der: Glucose ergab, dali $06 \%$ der eingeselaten thtivitit. an der ursprüglichen stelle (C-Alom 1) der (ilucose verblicben war. Th war dies der erste Vachwois oines zellfreien Finzrmsystems, das Hexosen zu Collulose polymerisieren ham. Bie Finergic fiir diese Reaktion entstammi der energiereichen Phosplarl. bindung des notwendigerweise zugesetaten Adenosintriphos. phates. In der anschliebenden Diskussion wurde die Mighlich. heit erörtert, dab vielleicht keine umnittelbare Polymerisation eintrete, sondern viehnehr erst cin Abbat der Glucose und warhfolgende Bilchung der Cellulose aus diesen Spaltstiichen.

In Vortrag von A. C. Neish Sashitoon, Canada) Bio synthesis of Hemicelluloses" wurden cinleitend die allen, sich teilweise widersprechenden Hypothesen über die Bio. synthese dieser Stoffklasse erwähnt. Gigene Versurhe des fortragenden bestäligen eine alte - und in der 7wischenteil wenig anerkannte - Hypothese (, $\mathrm{C}_{6}$-Deraboxyliomung" wonach Vorstufen der Pentosane aus l lexosen gebildet werden, welche das C.Atom 6 alspalten: Glucose- (1.4C) war bei Vorsuchen mit Weizen sowohl für die Cellulose als anch liir dis Xylan eine wirksame vorshate. Die nach dem Abban er. haltene Glucose und Xylose war vorwiegend joweils mur am C-Alom 1 aktir. Dic ebenfalls gratte Linwanllung ron Glucuronolacton in Xylan läbt die Beteiligung ciner L'Tronsäure an dieser Reaktionsfolge vermuten. Hingegen läßt sich freie Xyluse bei Weizen nicht zu Xylanen polynerisieren. Jis besteht Grund zur Annahme, daB Glycoside (Gridindiphosphatxylose die unmittelbaren Vorläufer der Pentosane darstellon. Ein ,Glycosid-Pool" , in dem Utridin-diphosphal glucose eine zontrale Stellung eimnimmt, unfabt zalalreiche Glycosyl. donatoren und soll allgemein mittels Transolvcosvlieruners. reaktionen lür die Biossuthese der Zellwandpolysaccharide verantworllich sein. An thand meturerer Beispicle wurde die Existenz des . Glycosid-Pools:", wie auch eines "Ester-Pools". der Zuckerphosphate umfißt und teilweise dem "GlycosidPool " vorgelagert ist; wabrscheinlich gemachl. 